\title{
Analysis of clinical characteristics and prognosis of talaromycosis (with or without human immunodeficiency virus) from a non-endemic area: a retrospective study
}

\author{
Qian Shen ${ }^{1} \cdot$ Lingyan Sheng $^{1} \cdot$ Junli Zhang ${ }^{2} \cdot \operatorname{Jian} \mathrm{Ye}^{3} \cdot$ Jianying Zhou $^{1}$
}

Received: 23 July 2020 / Accepted: 27 July 2021 / Published online: 5 August 2021

(c) Springer-Verlag GmbH Germany, part of Springer Nature 2021

\begin{abstract}
Purpose Talaromyces marneffei (TM) is a pathogenic fungus endemic in Southeast Asia and human immunodeficiency virus (HIV)-positive populations, but studies related to non-endemic areas and HIV-negative populations are still limited. Therefore, this study aims to provide more additional evidence for clinical work of talaromycosis.

Methods To collect clinical information of patients with talaromycosis admitted to hospitals in Zhejiang Province, China from January 1, 2010 to May 31, 2020, retrospectively analyzed clinical characteristics and prognosis, COX multivariate regression analysis was used for survival analysis.

Results A total of 92 patients were enrolled, including 76 males, 73 HIV-positive patients, with an average age of 40.1 \pm 13.0 . Compared to HIV-positive group, the negative group had higher admission age $(47.7 \pm 14.6$ vs $38.1 \pm 11.9, p=0.003)$ and lower proportion of male ( $89.0 \%$ vs $57.9 \%, p=0.004)$, there was no significant difference in imaging of lungs. There were significantly more HIV-positive patients in those with pleural effusion $(100 \%$ vs $69.4 \%, p=0.001)$. COX multivariate regression analysis suggested pleural effusion $(\mathrm{HR}=3.220 ; 95 \% \mathrm{CI} 1.117-9.287 ; p=0.030)$ and HIV infection $(\mathrm{HR}=0.057 ; 95 \%$ CI $0.009-0.370 ; p=0.003$ ) which were independent predictors of prognosis in patients with talaromycosis.

Conclusions In non-endemic areas, clinical symptoms, signs, and laboratory tests of patients with talaromycosis are similar to those in endemic areas. Patients with pleural effusion have lower survival rate, HIV-infected people are less likely to relapse, and there is no significant correlation between extent of lung involvement and survival of infected patients.
\end{abstract}

Keywords Talaromyces marneffei $\cdot$ Talaromycosis $\cdot$ Non-endemic Area $\cdot$ Human Immunodeficiency Virus $\cdot$ Clinical characteristics · Prognostic analysis

\section{Introduction}

Talaromyces marneffei (TM) is an opportunistic pathogenic fungus which can cause systemic disseminated infections.

Qian Shen and Lingyan Sheng contributed equally.

Jianying Zhou

zjyhz@zju.edu.cn

1 Department of Respiratory Medicine, The First Affiliated Hospital, School of Medicine, Zhejiang University, Hangzhou 310003, China

2 Department of Liver Infection, The Affiliated Sir Run Run Shaw Hospital, School of Medicine, Zhejiang University, Hangzhou 310016, China

3 Department of Respiratory Medicine, The Affiliated Hangzhou First People's Hospital, School of Medicine, Zhejiang University, Hangzhou 310003, China
It is the only known dimorphic fungus in Talaromyces at present $[1,2]$.

TM was originally isolated from a bamboo rat in Vietnam (1956) by Capponi and Sureau [3]. Since the first case of natural human infection reported in 1973 [4], more and more talaromycosis cases are reported. The vast majority of infected people are Human Immunodeficiency Virus/ Acquired Immunodeficiency Syndrome (HIV/AIDS) patients or other immunodeficiency patients with $\mathrm{CD}^{+}{ }^{+} \mathrm{T}$ cells $<100 / \mu \mathrm{L}$. Therefore, TM is endemic in Southeast Asia and South Asia, which are AIDS-endemic areas, mainly including Vietnam, India, Thailand, southern provinces of 
China (especially Guangxi, Guangdong) and Hong Kong of China [5-7].

Although talaromycosis is an endemic epidemic fungal disease, there are more and more infected patients in nonendemic areas and HIV-negative populations. Even HIVnegative infections with normal immune function have appeared [8]. However, studies on talaromycosis in nonendemic areas and HIV-negative populations are still limited. Therefore, more research is needed on people infected with TM (with or without HIV)in non-endemic areas.

In China, talaromycosis are mainly prevalent in individual southern provinces such as Guangdong, Guangxi, and Yunnan, and Zhejiang is a non-endemic province in East China. This study collected clinical information of patients with talaromycosis admitted to hospitals (mainly The First Affiliated Hospital, School of Medicine, Zhejiang University) in Zhejiang Province, China from January 1, 2010 to May 31, 2020 , then retrospectively analyzed and summarized their clinical characteristics, compared differences in clinical characteristics and prognosis of HIV-positive and negative patients, and compared against patients in endemic areas, which could provide more references and suggestions for related clinical diagnosis and treatment.

\section{Methods}

\section{Patients}

To collect clinical information of adult patients ( $\geq 18$ years old) from several hospitals in Zhejiang Province, China (mainly The First Affiliated Hospital, School of Medicine, Zhejiang University, the rest included The Affiliated Sir Run Run Shaw Hospital, School of Medicine, Zhejiang University, The Affiliated Hangzhou First People's Hospital, School of Medicine, Zhejiang University and Cangnan People's Hospital) with confirmed talaromycosis from January 1, 2010 to May 31, 2020. These patients were divided into two groups: HIV-positive and HIV-negative group.

Inclusion criteria: adult patients ( $\geq 18$ years of age) who had been determined to be infected with TM (with or without HIV) through pathogen culture or gene sequencing or microscope observation;

Exclusion criteria: patients with TM infections were determined by pathogen culture or gene sequencing or microscope observation, but it was not possible to determine whether they were HIV-infected.

Diagnostic criteria for talaromycosis:

1) Using standard culture techniques to isolate and culture TM from clinical specimens. Such clinical specimens mainly include blood, bone marrow, sputum, bronchoalveolar lavage fluid (BALF), skin secretions, skin biopsy specimens, cerebrospinal fluid, etc. $[9,10]$. The key evidence of the positive culture result is the thermal dimorphism of the pathogen, that is, the form of mycelium at $25^{\circ} \mathrm{C}$ coupling with brick red pigment and the form of yeast at $37^{\circ} \mathrm{C}$.

2) After Wright staining, Giemsa staining or periodic acid Schiff staining of cytology and histopathology specimens, the special yeast form and diaphragm of TM are directly observed with the microscope [11].

3) Polymerase Chain Reaction (PCR), Next Generation Sequencing (NGS) and other genetic testing methods specifically suggest that $\mathrm{TM}$ is positive.

\section{Clinical information}

Through the hospital's electronic information system, the patient's inpatient medical records were reviewed.

In this study, the patient's diagnosis of talaromycosis was mainly through pathogen culture and NGS. Pathogen culture and result judgment were completed by the hospital laboratory and NGS was carried out by sending out clinical specimens to specialized testing institutions. The imaging data used in this research all came from the hospital imaging system, and data were all based on patients' first noncontrast chest computed tomography (CT) after admission. The radiology reading work in the hospital was completed by the radiologist and subsequently reviewed by the respiratory physician.

The discharge outcome was defined as follows: (1) Effective: there was a stable and lasting recovery response after treatment with antifungal drugs. That was, the symptoms related to talaromycosis disappeared or the pathogen was negative again; (2) Recurrence: there was a temporary recovery response after treatment with antifungal drugs, but then symptoms related to talaromycosis appeared again, and the pathogen test was positive; (3) Death; (4) Lost to followup. Regarding the time of follow-up, all patients who did not have a death event were followed up to May 31, 2020, mainly through the patient's hospital visit records, hospitalization records and follow-up telephone calls.

\section{Statistical analysis}

All data in this study were statistically analyzed using SPSS 20.0 software (IBM SPSS Inc., Chicago, Illinois, USA). Continuous variables with normal distribution were expressed as mean \pm standard deviation (SD). The Kolmogorov-Smirnov test was used to determine whether the normal distribution condition was met. If $p>0.05$, then the normal distribution was met, and the $t$ test was used for comparison between groups. If the normal distribution was not met, non-parametric rank sum test was used for comparison between groups. The count data 
was expressed in terms of frequency and percentage, and Chi-square test or Fisher exact test was used for comparison between groups. Independent predictors related to prognosis were analyzed by COX multivariate regression analysis. All statistical analyses used a two-sided test and $p<0.05$ was considered statistically significant.

\section{Results}

A total of 92 adult patients ( $\geq 18$ years old) with confirmed talaromycosis and basically complete clinical data from January 1, 2010 to May 31, 2020 were enrolled. There were 73 cases in the HIV-positive group and 19 cases in the HIVnegative group. Patients were mainly from The First Affiliated Hospital, School of Medicine, Zhejiang University, and 5 cases (two HIV-negative and three HIV-positive) were from The Affiliated Sir Run Run Shaw Hospital, School of Medicine, Zhejiang University, 2 cases were from The Affiliated Hangzhou First People's Hospital, School of Medicine, Zhejiang University, and one was from Cangnan People's Hospital. Among these patients, 6 cases were definitely with a history of exposure to TM endemic areas, others' permanent residences were nearly all in Zhejiang Province. But the travel history of some patients was unavailable.

\section{Clinical baseline information of patients with talaromycosis}

92 cases with talaromycosis had an admission age range of $18 \sim 74$ years old, with an average of $40.1 \pm 13.0$. The admission age of HIV-negative group was significantly higher than the HIV-positive group $(47.7 \pm 14.6$ vs $38.1 \pm 11.9$, $p=0.003)$. Of the 92 patients, 76 were male $(82.6 \%)$ and 16 were female $(17.4 \%)$. The proportion of male in the
HIV-positive group was significantly higher than HIV-negative group $(89.0 \%$ vs $57.9 \%, p=0.004)$ (Table 1$)$.

There were 56 patients $(60.9 \%)$ with other comorbidities, and the proportion of patients with comorbidities in the HIV-negative group was significantly higher than HIVpositive group $(84.2 \%$ vs $54.8 \%, p=0.019)$. The detailed comorbidities can be seen in Supplemental Table 1. HIVpositive patients themselves had varying degrees of immunodeficiency, while in HIV-negative group, there was one patient each $(6.3 \%)$ with liver or kidney transplantation, two patients (12.5\%) with rheumatoid arthritis and one patient (6.3\%) with systemic lupus erythematosus (SLE).

With regard to the clinical symptoms and signs of TM infected people (Table 2), the most common clinical signs and symptoms of 92 patients were fever ( 75 cases, $81.5 \%$ ), lymphadenopathy (74 cases, $80.4 \%$ ) and cough (58 cases, $63.0 \%)$. Specifically, the proportions of patients with cough ( $84.2 \%$ vs $57.5 \%, p=0.032$ ), expectoration ( $78.9 \%$ vs $38.4 \%$, $p=0.002)$ and abnormal pulmonary breathing sounds (73.7\% vs $46.6 \%, p=0.035)$ in the HIV-negative group were significantly higher than HIV-positive group, and the proportion of fever $(86.3 \%$ vs $63.2 \%, p=0.047)$ and splenomegaly $(58.9 \%$ vs $10.5 \%, p<0.001)$ in the HIV-positive group was significantly higher than the HIV-negative group.

\section{Laboratory tests and chest CT results of patients with talaromycosis}

In terms of laboratory test results (Table 3 ), the counts of white blood cell (WBC) $(10.7 \pm 8.4$ vs $4.1 \pm 2.7, p=0.003)$, lymphocytes $(1.4 \pm 1.1$ vs $0.4 \pm 0.4, p<0.001)$ and platelets (PLT) $(270.3 \pm 189.2$ vs $121.4 \pm 104.7, p=0.001)$ in HIV-negative group were significantly higher than HIVpositive group. The average values of these three indicators were all much lower than normal. Moreover, alanine aminotransferase (ALT) $(66.8 \pm 71.2$ vs $34.3 \pm 36.4, p=0.016)$ and aspartate aminotransferase (AST) $(140.9 \pm 176.2 \mathrm{vs}$
Table 1 Clinical baseline data of patients infected with TM

\begin{tabular}{lllll}
\hline Items & $\begin{array}{l}\text { All } \\
n(\%)\end{array}$ & $\begin{array}{l}\text { HIV-positive } \\
n(\%)\end{array}$ & $\begin{array}{l}\text { HIV-negative } \\
n(\%)\end{array}$ & $p$ value \\
\hline Cases & 92 & 73 & 19 & $/$ \\
Admission age & $40.1 \pm 13.0$ & $38.1 \pm 11.9$ & $47.7 \pm 14.6$ & 0.003 \\
Age range & $18-74$ & $18-74$ & $24-72$ & $/$ \\
Male & $76(82.6)$ & $65(89.0)$ & $11(57.9)$ & 0.004 \\
Occupation & $86(93.5)$ & $69(94.5)$ & $17(89.5)$ & 0.786 \\
Smoking history & $22(23.9)$ & $17(23.3)$ & $5(26.3)$ & 1.000 \\
Drinking history & $14(15.2)$ & $12(16.4)$ & $2(10.5)$ & 0.779 \\
Comorbidity & $56(60.9)$ & $40(54.8)$ & $16(84.2)$ & 0.019 \\
History of co-infection & $77(83.7)$ & $60(82.2)$ & $17(89.5)$ & 0.677 \\
History of immunosuppressive & $14(15.2)$ & $5(6.8)$ & $9(47.4)$ & $<0.001$ \\
\multicolumn{1}{c}{ agents or hormone use } & & & & \\
\hline
\end{tabular}


Table 2 Clinical symptoms and signs of patients infected with TM

\begin{tabular}{|c|c|c|c|c|}
\hline Items & $\begin{array}{l}\text { All } \\
n(\%)\end{array}$ & $\begin{array}{l}\text { HIV-positive } \\
n(\%)\end{array}$ & $\begin{array}{l}\text { HIV-negative } \\
n(\%)\end{array}$ & $p$ value \\
\hline Cases & 92 & 73 & 19 & l \\
\hline Fever & $75(81.5)$ & $63(86.3)$ & $12(63.2)$ & 0.047 \\
\hline \multicolumn{5}{|l|}{ Severity of fever } \\
\hline Low & $8(10.7)$ & $5(7.9)$ & $3(25.0)$ & l \\
\hline Medium & $16(21.3)$ & $14(22.2)$ & $2(16.7)$ & I \\
\hline High & $38(50.7)$ & $33(52.4)$ & $5(41.6)$ & / \\
\hline Unknown & $13(17.3)$ & $11(17.5)$ & $2(16.7)$ & / \\
\hline \multicolumn{5}{|l|}{ Respiratory symptom } \\
\hline Cough & $58(63.0)$ & $42(57.5)$ & $16(84.2)$ & 0.032 \\
\hline Expectoration & $43(46.7)$ & $28(38.4)$ & $15(78.9)$ & 0.002 \\
\hline Dyspnea & 18 (19.6) & $13(17.8)$ & $5(26.3)$ & 0.611 \\
\hline \multicolumn{5}{|l|}{ Gastrointestinal symptom } \\
\hline Abdominal pain & $14(15.2)$ & $13(17.8)$ & $1(5.3)$ & 0.318 \\
\hline Diarrhea & $17(18.5)$ & $14(19.2)$ & $3(15.8)$ & 0.994 \\
\hline Weight loss & $41(44.6)$ & $36(49.3)$ & $5(26.3)$ & 0.072 \\
\hline Skin lesions & 45 (48.9) & $39(53.4)$ & $6(31.6)$ & 0.090 \\
\hline Bone and joint pain & $3(3.3)$ & $2(2.7)$ & $1(5.3)$ & 1.000 \\
\hline Lymphadenopathy & $74(80.4)$ & $58(79.5)$ & $16(84.2)$ & 0.888 \\
\hline Hepatomegaly & $7(7.6)$ & $7(9.6)$ & 0 & 0.358 \\
\hline Splenomegaly & $45(48.9)$ & $43(58.9)$ & $2(10.5)$ & $<0.001$ \\
\hline $\begin{array}{l}\text { Abnormal pulmonary breath- } \\
\text { ing sounds }\end{array}$ & $48(52.2)$ & $34(46.6)$ & $14(73.7)$ & 0.035 \\
\hline
\end{tabular}

Low: $\sim 38^{\circ} \mathrm{C}$; Medium: $\sim 39^{\circ} \mathrm{C}$; High: $\sim 41^{\circ} \mathrm{C}$

The proportion of fever with different degrees is the percentage of fever cases

\begin{tabular}{|c|c|c|c|c|}
\hline Items & $\begin{array}{l}\text { All } \\
n(\%)\end{array}$ & $\begin{array}{l}\text { HIV-positive } \\
n(\%)\end{array}$ & $\begin{array}{l}\text { HIV-negative } \\
n(\%)\end{array}$ & $p$ value \\
\hline Cases & 92 & 73 & 19 & l \\
\hline $\mathrm{Hb}(\mathrm{g} / \mathrm{L})$ & $98.1 \pm 21.4$ & $97.8 \pm 20.4$ & $99.2 \pm 25.2$ & 0.797 \\
\hline $\mathrm{WBC}\left(\times 10^{9} / \mathrm{L}\right)$ & $5.5 \pm 5.2$ & $4.1 \pm 2.7$ & $10.7 \pm 8.4$ & 0.003 \\
\hline Lymphocytes $\left(\times 10^{9} / \mathrm{L}\right)$ & $0.6 \pm 0.7$ & $0.4 \pm 0.4$ & $1.4 \pm 1.1$ & $<0.001$ \\
\hline Lymphocytes (\%) & $12.8 \pm 9.5$ & $11.8 \pm 8.7$ & $16.4 \pm 11.8$ & 0.104 \\
\hline Neutrophils (\%) & $79.9 \pm 12.7$ & $81.2 \pm 11.6$ & $75.2 \pm 15.8$ & 0.068 \\
\hline $\operatorname{PLT}\left(\times 10^{9} / \mathrm{L}\right)$ & $152.2 \pm 139.4$ & $121.4 \pm 104.7$ & $270.3 \pm 189.2$ & 0.001 \\
\hline Creatine $(\mu \mathrm{mol} / \mathrm{L})$ & $75.9 \pm 76.6$ & $71.7 \pm 38.1$ & $92.1 \pm 152.6$ & 0.103 \\
\hline ALT (U/L) & $60.1 \pm 66.7$ & $66.8 \pm 71.2$ & $34.3 \pm 36.4$ & 0.016 \\
\hline AST (U/L) & $123.2+163.8$ & $140.9 \pm 176.2$ & $55.1 \pm 72.6$ & 0.041 \\
\hline Albumin $(\mathrm{g} / \mathrm{L})$ & $28.0 \pm 6.1$ & $27.8 \pm 5.9$ & $28.9 \pm 6.9$ & 0.502 \\
\hline $\operatorname{ALP}(\mathrm{U} / \mathrm{L})$ & $186.9 \pm 218.6$ & $191.9 \pm 233.8$ & $167.6 \pm 150.2$ & 0.668 \\
\hline $\mathrm{CRP}(\mathrm{mg} / \mathrm{L})$ & $75.5 \pm 62.3$ & $73.7 \pm 61.1$ & $83.1 \pm 68.9$ & 0.579 \\
\hline $\operatorname{ESR}(\mathrm{mm} / \mathrm{h})$ & $53.0 \pm 33.9$ & $52.8 \pm 33.9$ & $54.1 \pm 35.0$ & 0.903 \\
\hline CD4+ T cells $(\%)$ & $10.8 \pm 13.7$ & $5.5 \pm 6.4$ & $34.9 \pm 12.1$ & $<0.001$ \\
\hline
\end{tabular}

$H b$ hemoglobin, $A L P$ alkaline phosphatase, ESR erythrocyte sedimentation rate
Table 3 Laboratory tests of patients infected with TM
$55.1 \pm 72.6, p=0.041)$ in the HIV-positive group were significantly higher than HIV-negative group. The percentage of $\mathrm{CD}^{+} \mathrm{T}$ cells was $5.5 \pm 6.4(\%)$ in the HIV-positive group $(n=59)$ and $34.9 \pm 12.1(\%)$ in the negative group $(n=13)$. There was a significant difference between the two groups $(p<0.001)$. 
In the chest CT images of 92 patients (Table 4), there were two in the HIV-positive group who had completely normal images at the time of admission. The remaining 90 patients all had abnormalities of varying degrees and types, and the typical chest $\mathrm{CT}$ images of several abnormal types could be seen in Fig. 1. Among them, high-density plaques/stripes ( 70 cases, $77.8 \%$ ), pulmonary nodules (59 cases, $65.6 \%$ ), hilar/mediastinal lymphadenopathy (47 cases, $52.2 \%$ ) and pleural effusion (30 cases, $33.3 \%$ ) were the most common presentations. There were no significant differences between the HIV-positive group and the HIV-negative group in all kinds of abnormal changes and extent of lung involvement $(p>0.05)$.

\section{Diagnosis and treatment of patients with talaromycosis}

92 patients included in this study all identified talaromycosis by means of pathogen culture or NGS, a total of 6 cases $(6.5 \%)$ were diagnosed by NGS, and the remaining 86 cases $(93.5 \%)$ were diagnosed by traditional pathogen culture, details can be seen in Supplemental Table 2. In terms of diagnostic interval, the average time of 89 patients was $8.4 \pm 5.5$ days, and there was no significant difference between two groups $(7.7 \pm 3.8$ vs $11.3 \pm 9.1, p>0.05)$.

Regarding the treatment plan (Table 5), except for one patient in each of the two groups who were discharged from the hospital without any treatment, the remaining 90 patients all received antifungal treatment. It was found that the proportion with selecting amphotericin B $(11.1 \%$ vs $0, p=0.049$ ) or voriconazole ( $44.4 \%$ vs $12.5 \%, p=0.006$ ) alone in HIV-negative group was significantly higher than
Table 4 Chest CT images among patients infected with TM

\begin{tabular}{|c|c|c|c|c|}
\hline Items & $\begin{array}{l}\text { All } \\
n(\%)\end{array}$ & $\begin{array}{l}\text { HIV-positive } \\
n(\%)\end{array}$ & $\begin{array}{l}\text { HIV-negative } \\
n(\%)\end{array}$ & $p$ value \\
\hline Cases & 92 & 73 & 19 & / \\
\hline Normal & $2(2.2)$ & $2(2.7)$ & 0 & 1.000 \\
\hline Abnormal & $90(97.8)$ & $71(97.3)$ & $19(100)$ & \\
\hline \multicolumn{5}{|l|}{ Abnormal types } \\
\hline Unilateral & $15(16.7)$ & $13(18.3)$ & $2(10.5)$ & 0.644 \\
\hline Nodule & $59(65.6)$ & $46(64.8)$ & $13(68.4)$ & 0.767 \\
\hline High-density plaque/strip & $70(77.8)$ & $52(73.2)$ & $18(94.7)$ & 0.091 \\
\hline Cavity & $8(8.9)$ & $4(5.6)$ & $4(21.1)$ & 0.100 \\
\hline Mass & $3(3.3)$ & $3(4.2)$ & 0 & 0.848 \\
\hline Interstitial lesions & $1(1.1)$ & $1(1.4)$ & 0 & 1.000 \\
\hline Pleural effusion & $30(33.3)$ & $21(29.6)$ & $9(47.4)$ & 0.144 \\
\hline Pericardial effusion & $11(12.2)$ & $10(14.1)$ & $1(5.3)$ & 0.517 \\
\hline Hilar/mediastinum lymphadenopathy & $47(52.2)$ & $35(49.3)$ & $12(63.2)$ & 0.283 \\
\hline Emphysema & $15(16.7)$ & $12(16.9)$ & $3(15.8)$ & 1.000 \\
\hline
\end{tabular}

The percentage of abnormal manifestations of lung CT imaging was the percentage of abnormal cases
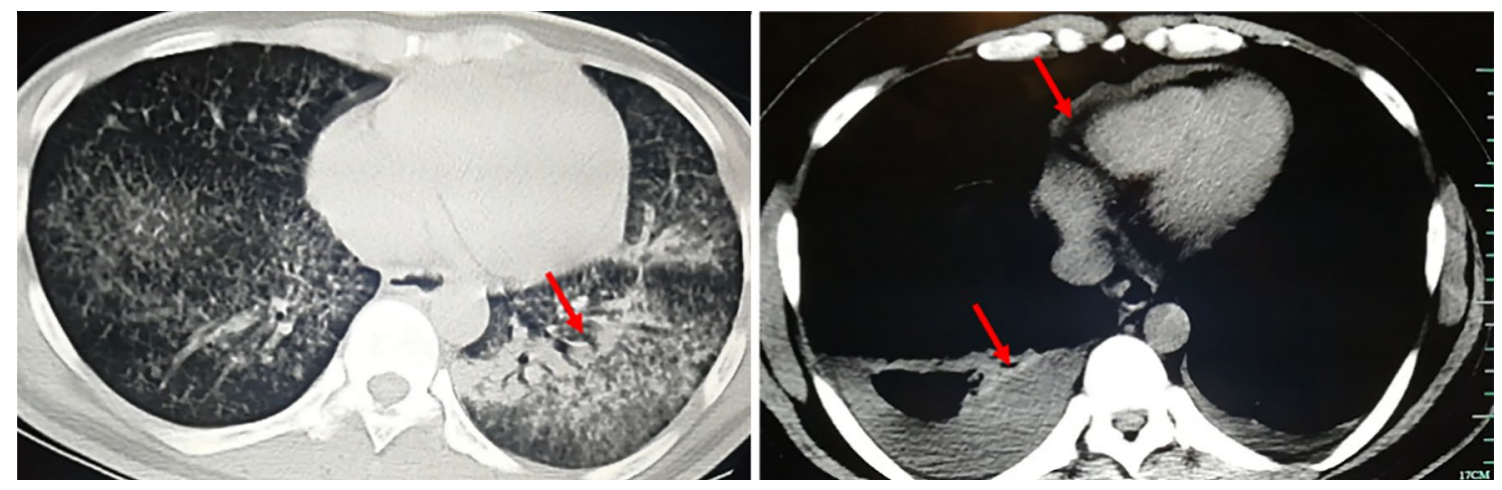

Fig. 1 Chest CT images among patients infected with TM (from left to right: high-density plaque/strip; pleural effusion + pericardial effusion) 
Table 5 Treatment Schemes for patients infected with TM

\begin{tabular}{lllll}
\hline Items & $\begin{array}{l}\text { All } \\
n(\%)\end{array}$ & $\begin{array}{l}\text { HIV-positive } \\
n(\%)\end{array}$ & $\begin{array}{l}\text { HIV-negative } \\
n(\%)\end{array}$ & $p$ value \\
\hline Cases & 92 & 73 & 19 & $/$ \\
Cases with antifungal treatment & $90(97.8)$ & $72(98.6)$ & $18(94.7)$ & 0.878 \\
Fluconazole & $4(4.4)$ & $2(2.8)$ & $2(11.1)$ & 0.371 \\
Amphotericin B & $2(2.2)$ & 0 & $2(11.1)$ & 0.049 \\
Itraconazole & $4(4.4)$ & $2(2.8)$ & $2(11.1)$ & 0.371 \\
Voriconazole & $17(18.9)$ & $9(12.5)$ & $8(44.4)$ & 0.006 \\
Amphotericin B + Voriconazole & $17(18.9)$ & $16(22.2)$ & $1(5.6)$ & 0.201 \\
Amphotericin B + Itraconazole & $21(23.3)$ & $20(27.8)$ & $1(5.6)$ & 0.093 \\
Voriconazole + Itraconazole & $2(2.2)$ & $2(2.8)$ & 0 & 1.000 \\
Amphotericin B + Itraconazole + Voriconazole & $13(14.4)$ & $12(16.7)$ & $1(5.6)$ & 0.410 \\
Amphotericin B + Itraconazole + Voricona- & $1(1.1)$ & $1(1.4)$ & 0 & 1.000 \\
$\quad$ zole+Fluconazole & & & & 0.792 \\
Others & $9(10.0)$ & $8(11.1)$ & $1(5.6)$ & \\
\hline
\end{tabular}

Others: In the HIV-positive group, including 2 cases of fluconazole + amphotericin B, 1 cases each of fluconazole + voriconazole/nystatin/itraconazole, 1 case of nystatin, 1 case each of amphotericin B + fluconazole + voriconazole/itraconazole; in HIV-negative group, including 1 case caspofungin
HIV-positive group, and there was no significant difference between two groups in the remaining treatment options $(p>0.05)$.

The average hospital stay of 92 patients was $20.0 \pm 10.9$ days, and there was no significant difference in the length of hospitalization between two groups $(19.7 \pm 10.3$ vs $20.9 \pm 13.0, p>0.05)$. The total number of lost to followup was 13 cases (14.1\%), including 11 cases in the HIV-positive group (15.1\%) and 2 cases in the HIV-negative group $(10.5 \%)$. The lost to follow-up rate in both groups reached more than $10 \%$, and the effective rate of treatment in the HIV-positive group was significantly higher than that in the HIV-negative group (77.4\% vs $41.2 \%, p=0.004)$ (Table 6).

\section{Clinical characteristics and prognosis of patients with talaromycosis under different imaging characteristics}

The data of chest CT images in this study were all based on patients' first non-contrast chest CT after admission, it is meaningful to explore clinical characteristics and prognosis of patients with talaromycosis under different imaging characteristics. When calculating the prognostic-related differences between groups, relapse-related analysis removed both lost and dead cases, and the rest only removed lost to follow-up cases.

In the unilateral/bilateral lung involvement groups (Table 7), there were 90 patients with lung involvement, 15 patients were with unilateral involvement $(16.7 \%)$, and 75 patients were with bilateral involvement $(63.3 \%)$. There was no significant difference in clinical symptoms and signs, laboratory test results and chest CT images between two groups $(p>0.05)$. But the recurrence rate of patients with unilateral
Table 6 Follow-up of patients infected with TM

\begin{tabular}{lllll}
\hline Items & $\begin{array}{l}\text { All } \\
n(\%)\end{array}$ & $\begin{array}{l}\text { HIV-positive } \\
n(\%)\end{array}$ & $\begin{array}{l}\text { HIV-negative } \\
n(\%)\end{array}$ & $p$ value \\
\hline $\begin{array}{l}\text { Cases } \\
\text { Discharge outcome }\end{array}$ & 92 & 73 & 19 & $/$ \\
Effective & $55(69.6)$ & $48(77.4)$ & $7(41.2)$ & 0.004 \\
Recurrence & $10(12.7)$ & $6(9.7)$ & $4(23.5)$ & 0.097 \\
Death & $14(17.7)$ & $8(12.9)$ & $6(35.3)$ & 0.075 \\
Lost to follow-up & $13(14.1)$ & $11(15.1)$ & $2(10.5)$ & 0.891 \\
Length of hospital stay/days & $20.0 \pm 10.9$ & $19.7 \pm 10.3$ & $20.9 \pm 13.0$ & 0.683 \\
\hline
\end{tabular}

When calculating the prognostic-related differences between two groups, relapse-related analysis removed both lost and dead cases, and the rest only removed lost to follow-up cases 
Table 7 Corresponding clinical characteristics and prognosis in patients with unilateral/bilateral lung involvement

\begin{tabular}{|c|c|c|c|c|}
\hline Items & $\begin{array}{l}\text { All } \\
n(\%)\end{array}$ & $\begin{array}{l}\text { Unilateral } \\
n(\%)\end{array}$ & $\begin{array}{l}\text { Bilateral } \\
n(\%)\end{array}$ & $p$ value \\
\hline Cases & 90 & 15 & 75 & I \\
\hline Admission age & $40.4 \pm 13.0$ & $37.7 \pm 9.7$ & $40.9 \pm 13.5$ & 0.389 \\
\hline Male & $74(82.2)$ & $10(66.7)$ & $64(85.3)$ & 0.175 \\
\hline HIV infection & $71(78.9)$ & $13(86.7)$ & $58(77.3)$ & 0.644 \\
\hline Smoking history & $22(24.4)$ & $2(13.3)$ & $20(26.7)$ & 0.443 \\
\hline Drinking history & $14(15.6)$ & $2(13.3)$ & $12(16.0)$ & 1.000 \\
\hline Comorbidity & $53(58.9)$ & $9(60.0)$ & $44(58.7)$ & 0.924 \\
\hline History of co-infection & $75(83.3)$ & $10(66.7)$ & $65(86.7)$ & 0.129 \\
\hline $\begin{array}{l}\text { History of immunosuppressive agents or } \\
\text { hormone use }\end{array}$ & $14(15.6)$ & $3(20.0)$ & $11(14.7)$ & 0.897 \\
\hline Fever & $73(81.1)$ & $12(80.0)$ & $61(81.3)$ & 1.000 \\
\hline \multicolumn{5}{|l|}{ Respiratory symptom } \\
\hline Cough & $56(62.2)$ & $9(60.0)$ & $47(62.7)$ & 0.846 \\
\hline Expectoration & $42(46.7)$ & $7(46.7)$ & $35(46.7)$ & 1.000 \\
\hline Dyspnea & $18(20.0)$ & $4(26.7)$ & $14(18.7)$ & 0.724 \\
\hline Skin lesions & $44(48.9)$ & $8(53.3)$ & $36(48.0)$ & 0.706 \\
\hline Lymphadenopathy & $73(81.1)$ & $11(73.3)$ & $62(82.7)$ & 0.630 \\
\hline Abnormal pulmonary breathing sounds & $47(52.2)$ & $6(40.0)$ & $41(54.7)$ & 0.299 \\
\hline Splenomegaly & $44(48.9)$ & $7(46.7)$ & $37(49.3)$ & 0.850 \\
\hline Hepatomegaly & $7(7.8)$ & $2(13.3)$ & $5(6.7)$ & 0.725 \\
\hline $\mathrm{WBC}\left(\times 10^{9} / \mathrm{L}\right)$ & $5.6 \pm 5.2$ & $5.5 \pm 6.3$ & $5.6 \pm 5.0$ & 0.953 \\
\hline Lymphocytes (\%) & $12.7 \pm 9.6$ & $15.9 \pm 11.7$ & $12.1 \pm 9.0$ & 0.153 \\
\hline Creatine $(\mu \mathrm{mol} / \mathrm{L})$ & $76.3 \pm 77.5$ & $76.3 \pm 51.9$ & $76.3 \pm 82.0$ & 0.999 \\
\hline Lymphocytes $\left(\times 10^{9} / \mathrm{L}\right)$ & $0.6 \pm 0.7$ & $0.7 \pm 0.5$ & $0.6 \pm 0.7$ & 0.766 \\
\hline $\operatorname{PLT}\left(\times 10^{9} / \mathrm{L}\right)$ & $153.7 \pm 140.5$ & $186.0 \pm 166.8$ & $147.3 \pm 135.0$ & 0.332 \\
\hline ALT (U/L) & $60.7 \pm 67.3$ & $54.9 \pm 38.8$ & $61.9 \pm 71.8$ & 0.716 \\
\hline AST (U/L) & $123.1 \pm 165.1$ & $96.1 \pm 83.9$ & $128.5 \pm 176.8$ & 0.492 \\
\hline Albumin $(\mathrm{g} / \mathrm{L})$ & $28.0 \pm 6.0$ & $30.2 \pm 6.9$ & $27.5 \pm 5.8$ & 0.123 \\
\hline ALP (U/L) & $188.6 \pm 220.7$ & $275.5 \pm 375.2$ & $171.2 \pm 173.5$ & 0.095 \\
\hline $\mathrm{CRP}(\mathrm{mg} / \mathrm{L})$ & $75.4 \pm 62.6$ & $63.0 \pm 61.3$ & $78.0 \pm 63.0$ & 0.401 \\
\hline $\mathrm{ESR}(\mathrm{mm} / \mathrm{h})$ & $53.6 \pm 34.1$ & $54.2 \pm 29.6$ & $53.4 \pm 35.2$ & 0.938 \\
\hline $\mathrm{CD} 4+\mathrm{T}$ cells $(\%)$ & $10.8 \pm 13.8$ & $10.1 \pm 13.1$ & $11.0 \pm 14.0$ & 0.848 \\
\hline Diagnostic interval/days & $8.4 \pm 5.5$ & $9.7 \pm 5.7$ & $8.2 \pm 5.5$ & 0.324 \\
\hline \multicolumn{5}{|l|}{ Discharge outcome } \\
\hline Effective & $54(69.2)$ & $7(53.8)$ & $47(72.3)$ & 0.323 \\
\hline Recurrence & $10(12.8)$ & $5(38.5)$ & $5(7.7)$ & 0.021 \\
\hline Death & $14(17.9)$ & $1(7.7)$ & $13(20.0)$ & 0.509 \\
\hline Lost to follow-up & $12(13.3)$ & $2(13.3)$ & $10(13.3)$ & 1.000 \\
\hline Length of hospital stay/days & $20.0 \pm 11.0$ & $23.4 \pm 12.5$ & $19.3 \pm 10.6$ & 0.190 \\
\hline
\end{tabular}

lung involvement was significantly higher than those with bilateral involvement $(38.5 \%$ vs $7.7 \%, p=0.021)$.

In the group with/without pleural effusion (Supplemental Table 3), a total of 92 cases were included in the analysis. A total of 30 patients $(32.6 \%)$ had pleural effusion and 60 patients $(67.4 \%)$ were without pleural effusion. The proportion of HIV-positive patients in patients with pleural effusion was significantly higher than those without pleural effusion (100\% vs $69.4 \%, p=0.001)$, and the albumin in laboratory results was lower $(24.5 \pm 5.4$ vs $29.7 \pm 5.6, p<0.001)$, the
C-reactive protein (CRP) value was higher $(94.3 \pm 63.7$ vs $66.1 \pm 60.0, p=0.043)$.

In the group with/without nodular lesions (Supplemental Table 4), a total of 92 cases were included in the analysis. There were 61 patients $(66.3 \%)$ with nodular lesions and 31 patients $(33.7 \%)$ without nodular lesions. Patients with nodular foci on lung imaging were more prone to have cough (70.5\% vs $90.2 \%, p<0.038)$ and surface lymphadenopathy (70.5\% vs $61.3 \%, p=0.001)$, while the incidence of dyspnea was lower $(13.1 \%$ vs $32.3 \%, p=0.029)$. 


\section{Survival analysis of patients with talaromycosis}

During the follow-up time of all patients, 11 cases were lost to follow-up in the HIV-positive group and 2 cases were in the HIV-negative group. The time span of this study was 10 years, so the lost cases were not included in the survival analysis.

In this study, COX regression analysis was used to determine the independent predictors of prognosis of talaromycosis. In all 92 patients, a total of 79 patients were included in this analysis, including 10 patients with recurrence $(12.7 \%)$ and 14 patients with death (17.7\%). In the study of independent predictive factors for patients' survival, factors with $p<0.1$ (HIV infection, comorbidities, diagnostic interval, lymphadenopathy, creatinine, nodules, pleural effusion) were included in multivariate regression by univariate regression analysis. The result showed that pleural effusion $(\mathrm{HR}=3.220 ; 95 \%$ CI: 1.117-9.287; $p=0.030$ ) was an independent predictor of survival in patients with talaromycosis. Similarly, it was concluded that HIV infection (HR $=0.057$; 95\% CI: 0.009-0.370; $p=0.003$ ) was an independent predictor of recurrence-free in patients with talaromycosis (Table 8).

\section{Discussion}

In this study, HIV/AIDS patients are still the main part of TM infected population in non-endemic area. Yongxuan $\mathrm{Hu}$ et al. [1] retrospectively analyzed 668 cases of talaromycosis reported in Mainland China from 1984 to 2009, and found that $99.4 \%$ of the cases were from cities in southern China, of which Guangxi accounted for $42.8 \%$ and Guangdong accounted for $40.6 \%, 87.7 \%$ of the cases were combined with HIV infection. It can be concluded that the distribution of talaromycosis in non-endemic areas is similar to the overall situation in endemic areas. Moreover, younger males from HIV-positive populations in non-endemic areas are the main population of talaromycosis, which is consistent with a previous literature reported in Thailand [12]. This conclusion is also in line with the current situation of HIV/AIDS prevalence among young males.

In China, as the migration population increases year by year, the disease has traveled far beyond the original areas like Guangdong and Guangxi. 21 other provinces and cities in China have reported cases [13]. This study included 6 cases with definite history of exposure to TM endemic areas, while most others are permanent residences of Zhejiang Province.
Therefore, it is justifiable that talaromycosis is no longer case in Zhejiang Province, and it is worthy of attention.

The comorbidities explored in this study did not show any primary immunodeficiency, but HIV-negative patients also included several cases with acquired immunodeficiency. These patients could suffer from severe immunodeficiency due to taking immunosuppressive agents or hormones, which is consistent with the results of previous studies [14, 15]. In HIV-negative populations, there were three patients without comorbidity. In addition to two patients with a history of tuberculosis, the others' immune function was completely normal and had no relevant history. Such patients are rare in talaromycosis, in 2018, a study suggested that only four cases of talaromycosis were reported in non-endemic areas. Therefore, future studies on genetics and other levels in this special population may need to be more in-depth to fully understand talaromycosis $[8,16]$.

The findings of clinical symptoms, signs, and laboratory tests in this study were basically consistent with the description of related research in endemic areas [12,17]. Some previous studies tried to explore the differences in clinical manifestations between HIV-positive and negative populations, but some small differences were usually ignored $[18,19]$. This study is the first large retrospective study in this area (nonendemic areas), the significant differences in clinical manifestations between the two groups could provide some constructive references for clinical work.

In this study, 92 patients were included to determine talaromycosis by pathogen culture or NGS, without serological diagnosis. Studies have shown that in practical clinical applications, the beta-D-glucan test and GM (Galactomannan) test have a certain role in monitoring and auxiliary diagnosis of talaromycosis [20,21]. They were applied in the treatment and follow-up of these patients. Previous study also showed that adult immunodeficiency caused by anti-IFN- $\gamma$ autoantibodies could explain many previous HIV-negative Asian infected patients. Since these patients had no remaining symbiotic diseases, whether anti-IFN- $\gamma$ autoantibodies could enter the clinic in the future as a screening indicator for TM infection in HIV-negative population is still to be studied [22]. In addition, an antibody test for TM Mp1p based on ELISA (Enzyme Linked Immunosorbent Assay) has been developed previously, and the results proved that about $80 \%$ of the HIV-positive TM infections could respond specifically [23], and there are still many research on serological diagnosis of TM. If the fungal infection can be directly diagnosed by serological testing in the
Table 8 COX multivariate regression analysis for the prognosis of people infected with TM

\begin{tabular}{llllll}
\hline Prognosis & Items & HR & \multicolumn{2}{l}{$95 \%$ Confidence Interval } & $P$ value \\
\cline { 4 - 5 } & & & Lower limit & Upper limit & \\
\hline Recurrence-free & HIV infection & 0.057 & 0.009 & 0.370 & 0.003 \\
Survival & Pleural effusion & 3.220 & 1.117 & 9.287 & 0.030 \\
\hline
\end{tabular}


future, there is no doubt that it is a milestone for the diagnosis of talaromycosis.

The imaging data in this study were all based on patients' first chest $\mathrm{CT}$ after admission, but the time intervals between admission and CT-scanning were not consistent. Previous studies have shown that in patients with talaromycosis in the lungs, the size of the lung lesions and pleural effusion are not associated with recurrence-free survival [24]. The result in this study was consistent with it. Moreover, when patients are suspected of being infected with TM and their lung images suggest the presence of pleural effusions, screening for HIV infection is required. Previous studies have explored pleural effusions of HIV-negative patients with talaromycosis. Laboratory analysis of hydrothorax showed that it was characterized by neutrophil-based light yellow pleural effusion, and high neutrophil count and IL-23 may prompt talaromycosis [25]. This can be considered in actual work based on pleural effusion as an auxiliary reference for diagnosis of HIV-positive talaromycosis.

Previous study suggested that the total mortality rate of talaromycosis in endemic areas is $22.7 \%$ [12]. In this study, the mortality rate was $15.2 \%$ in the existing information, which was lower than the value reported in the literature, despite certain censored data. But this may also be related to factors such as strict supervision of HIV-positive people. This study suggests that pleural effusion is related to patient's survival, there is no similar study before, and the follow-up can be continued to explore the reliability and stability of this conclusion by increasing the sample size. In actual clinical work, patients with pleural effusion should be alert to the possibility of death and follow-up should be strengthened. This study suggests that the recurrence rate of HIV-positive patients is lower. This may be related to the strict supervision of these patients. People with HIVnegative infection often have secondary immunodeficiency, more comorbidities, and poor basic conditions, which may also be related to this result.

Of course, this study still has some limitations. Firstly, the number of infections is still not comparable with those in the endemic areas, which may prevent some variables from being revealed. Secondly, all patients have a hospital admission span of up to 10 years, there is a hospital bias, and related laboratory testing techniques are different in each time period. Some data are missing, such as whether the patient has a history of exposure to TM endemic areas. In addition, most of the patients in this study are HIV/AIDS patients, and there are certain limitations of follow-up due to objective reasons such as privacy protection. Finally, because this study is a retrospective study, some of the correlations mentioned in the text can only provide as references. However, as the first large retrospective study in Zhejiang Province, it still attracts attention to talaromycosis in endemic areas and provides several practical suggestions for clinical work, and it is also necessary to increase sample size further to make conclusions more convincing.

Supplementary Information The online version contains supplementary material available at https://doi.org/10.1007/s15010-021-01679-6.

Acknowledgements We acknowledge The Affiliated Hangzhou First People's Hospital, School of Medicine, Zhejiang University, The Affiliated Sir Run Run Shaw Hospital, School of Medicine, Zhejiang University and Cangnan People's Hospital for providing partial data

Author contribution SQ and ZJY conceived the ideas; SLY, ZJL and YJ collected the data; SLY and SQ analyzed the data and wrote manuscript; SQ and ZJY led the writing and reviewing.

Funding Not applicable.

Availability of data and material Data and material are submitted in this study and electronic supplementary material.

Code availability Not applicable.

\section{Declarations}

Conflict of interest Not applicable.

Ethics approval The authors confirm that the ethical policies of the journal, as noted on the journal's author guidelines page, have been adhered to and the appropriate ethical review committee approval has been received.

Consent for participate Not applicable.

Consent for publication Informed consent was obtained from patients for publication of this report and accompanying images.

\section{References}

1. Hu Y, Zhang J, Li X, Yang Y, Zhang Y, Ma J, et al. Penicillium marneffei infection: an emerging disease in mainland China. Mycopathologia. 2013;175:57-67.

2. Duong TA. Infection due to Penicillium marneffei, an emerging pathogen: review of 155 reported cases. Clin Infect Dis. 1996;23:125-30.

3. Capponi M, Segretain G, Sureau P. Penicillosis from Rhizomys sinensis. Bull de la Soc de Pathol Exotique et de ses Filiales. 1956;49:418-21.

4. DiSalvo AF, Fickling AM, Ajello L. Infection caused by Penicillium marneffei: description of first natural infection in man. Am J Clin Pathol. 1973;60:259-63.

5. Li HR, Cai SX, Chen YS, Yu ME, Xu NL, Xie BS, et al. Comparison of Talaromyces marneffei infection in human immunodeficiency virus-positive and human immunodeficiency virus-negative patients from Fujian, China. Chin Med J Peking. 2016;129:1059-65.

6. Ustianowski AP, Sieu TP, Day JN. Penicillium marneffei infection in HIV. Curr Opin Infect Dis. 2008;21:31-6.

7. Yuen KY, Wong SS, Tsang DN, Chau PY. Serodiagnosis of Penicillium marneffei infection. Lancet (Lond, Engl). 1994;344:444-5.

8. Castro-Lainez MT, Sierra-Hoffman M, Lompant-Zeno J, Adams R, Howell A, Hoffman-Roberts H, et al. Talaromyces marneffei 
infection in a non-HIV non-endemic population. IDCases. 2018;12:21-4.

9. Le T, Kinh NV, Cuc NTK, Tung NLN, Lam NT, Thuy PTT, et al. A trial of itraconazole or amphotericin B for HIV-associated talaromycosis. New Engl J Med. 2017;376:2329-40.

10. Zeng W, Qiu Y, Lu D, Zhang J, Zhong X, Liu G. A Retrospective analysis of 7 human immunodeficiency virus-negative infants infected by Penicillium marneffei. Med. 2015;94:1439.

11. Jan IS, Chung PF, Wang JY, Weng MH, Hung CC, Lee LN. Cytological diagnosis of Penicillium marneffei infection. J Formos Med Assoc Taiwan Yi Zhi. 2008;107:443-7.

12. Kawila R, Chaiwarith R, Supparatpinyo K. Clinical and laboratory characteristics of Penicilliosis marneffei among patients with and without HIV infection in Northern Thailand: a retrospective study. BMC Infect Dis. 2013;13:464.

13. Cao C, Xi L, Chaturvedi V. Talaromycosis (Penicilliosis) Due to Talaromyces (Penicillium) marneffei: insights into the clinical trends of a major fungal disease 60 years after the discovery of the pathogen. Mycopathologia. 2019;184:709-20.

14. Lee PP, Chan KW, Lee TL, Ho MH, Chen XY, Li CH, et al. Penicilliosis in children without HIV infection-are they immunodeficient? Clin Infect Dis. 2012;54:e8-19.

15. Luo DQ, Chen MC, Liu JH, Li Z, Li HT. Disseminated Penicillium marneffei infection in an SLE patient: a case report and literature review. Mycopathologia. 2011;171:191-6.

16. Saadiah S, Jeffrey AH, Mohamed AL. Penicillium marneffei infection in a non aids patient: first case report from Malaysia. Med J Malaysia. 1999;54:264-6.

17. Supparatpinyo K, Chiewchanvit S, Hirunsri P, Uthammachai C, Nelson KE, Sirisanthana T. Penicillium marneffei infection in patients infected with human immunodeficiency virus. Clin Infect Dis. 1992;14:871-4.

18. Wong SY, Wong KF. Penicillium marneffei Infection in AIDS. Pathol Res Int. 2011;2011:764293.
19. Zhang JQ, Yang ML, Zhong XN, He ZY, Liu GN, Deng JM, et al. A comparative analysis of the clinical and laboratory characteristics in disseminated penicilliosis marneffei in patients with and without human immunodeficiency virus infection. Zhonghua Jie He He Hu Xi Za Zhi Zhonghua Jiehe He Huxi Zazhi Chin J Tuberc Respir Dis. 2008;31:740-6.

20. Yoshimura Y, Sakamoto Y, Lee K, Amano Y, Tachikawa N. Penicillium marneffei infection with $\beta$-D -glucan elevation: a case report and literature review. Internal Med (Tokyo, Japan). 2016;55:2503-6.

21. Huang YT, Hung CC, Liao CH, Sun HY, Chang SC, Chen YC. Detection of circulating galactomannan in serum samples for diagnosis of Penicillium marneffei infection and cryptococcosis among patients infected with human immunodeficiency virus. J Clin Microbiol. 2007;45:2858-62.

22. Tang BS, Chan JF, Chen M, Tsang OT, Mok MY, Lai RW, et al. Disseminated penicilliosis, recurrent bacteremic nontyphoidal salmonellosis, and burkholderiosis associated with acquired immunodeficiency due to autoantibody against gamma interferon. Clin Vaccine Immunol. 2010;17:1132-8.

23. Cao L, Chen DL, Lee C, Chan CM, Chan KM, Vanittanakom N, et al. Detection of specific antibodies to an antigenic mannoprotein for diagnosis of Penicillium marneffei penicilliosis. J Clin Microbiol. 1998;36:3028-31.

24. Qiu Y, Zhang JQ, Pan ML, Zeng W, Tang SD, Tan CM. Determinants of prognosis in Talaromyces marneffei infections with respiratory system lesions. Chin Med J Peking. 2019;132:1909-18.

25. Qiu Y, Zeng W, Zhang H, Zhong X, Tang S, Zhang J. Comparison of pleural effusion features and biomarkers between talaromycosis and tuberculosis in non-human immunodeficiency virus-infected patients. BMC Infect Dis. 2019;19:745. 